\title{
Modelo para mapeamento das percepções dos usuários a respeito dos serviços prestados por uma biblioteca especializada
}

Maria Bernarda Teixeira Duarte

Graduada em Bilbioteconomia e Documentação pela UNIRIO.Mestre em Sistemas de Gestão pela UFF

Helder Gomes Costa

Graduado em Engenharia Mecânica pela Universidade Federal Fluminense. Mestre e doutor em Engenharia Mecânica pela PUC-Rio,. Professor Titular na UFF e pesquisador em Produtividade em Pesquisa pelo CNPq

http://dx.doi.org/10.1590/1981-5344/2463

No ambiente da gestão de bibliotecas, vem à tona a seguinte questão: como avaliar a satisfação dos usuários com os serviços prestados pelas bibliotecas? Este artigo propõe e aplica um modelo para a avaliação da satisfação dos usuários quanto aos serviços oferecidos por uma biblioteca e, também, identificar o grau de importância dos diferentes aspectos deste tipo de serviço. Para isto uma revisão sistematizada da literatura foi desenvolvida a fim de dar suporte à construção do modelo. Esta revisão foi especialmente útil para definir o conjunto de critérios de avaliação e para a elaboração do instrumento de coleta, o qual foi aplicado a uma amostra composta por 117 usuários de uma biblioteca especializada na área de tecnologia nuclear, dos quais retornaram 94 formulários. Os dados coletados indicaram a importância e o desempenho de cada um dos aspectos considerados no questionário, apontando os critérios nos quais os usuários ficaram mais satisfeitos foram a conservação e a adequação do acervo. Por outro lado, os resultados também mostraram que atualização e cobertura internacional do acervo são os aspectos prioritários para ações de melhoria, visto que são aqueles em que os usuários estão menos satisfeitos e também são aqueles que os usuários consideram mais importantes. Destacamse como contribuições da pesquisa: o conjunto de 
critérios; o instrumento de coleta; e, a proposta para tratamento dos dados.

Palavras-chave: Avaliação de serviços; Serviços de biblioteca; Qualidade dos serviços.

\section{A model for mapping users perceptions about the services supplied by a specialized library}

Nowadays a question arises: how to evaluate the users' satisfaction regarding the services provided by a library? This paper aims to propose and apply a model for evaluating the satisfaction of the users of a library, regarding the library services. The proposal should be divided into three steps: a systematized literature review that has supported the construction of a questionnaire; a data collection though applying the questionnaire to a sample; and, the analysis of the data collected. In the first step, it was performed an extensive and systematized literature review on the bases Scientific Electronic Library Online - SciELO, Scopus and ISI Web of Science was developed to support the construction this model. The results from the literature review were the input for defining the set of evaluation criteria and to prepare a questionnaire for evaluating the users' satisfaction. In the second step the questionnaire was applied to a sample by users of a library specialized in the field of nuclear technology, in Rio de Janeiro Brazil. In the third step, the data collected were complied and analysed though statistical frequency analysis. The sample was composed of 117 respondents of whom 94 returned forms. The results indicated that the criteria on which the library best performed were the conservation and adequacy of the collection. In the other hand, the results also have shown that the update criteria and international coverage of the collection are the ones that have should receive more attention, once they were perceived as having low performance and high importance for the users' satisfaction. The main contributions of this research are: the criteria set and the frame for performing the survey and the way data were analysed. 
Keywords: Services' evaluation; Library services; Quality of Services.

Recebido em 28.07.2015 Aceito em 17.04.2017

\section{Introdução}

A construção de modelos para avaliação de serviços prestados por bibliotecas é um processo dinâmico e em constante atualização, o que é causado principalmente pela constante inovação nas formas de acesso de disponibilização à informação. Este fato implica na necessidade de revisão e atualização dos critérios de avaliação ao se construir um modelo para avaliação de serviços deste tipo.

Neste sentido, faz-se necessária à realização de um levantamento de critérios de avaliação de serviços e sua adequação ao contexto de bibliotecas. Apesar da existência de uma grande quantidade de trabalhos prévios de pesquisa para avaliação deste tipo de serviço, as questões de pesquisa descritas a seguir permanecem em aberto ou demandam atualização de suas respostas, devendo ser respondidas no contexto desta pesquisa. Neste contexto, surgem as seguintes questões:

a)Quais seriam os critérios para avaliação das percepções de usuário de uma biblioteca especializada, quanto aos serviços prestados pela mesma?

b)Como captar e tratar estas percepções?

c)Quais seriam estas percepções no caso específico dos usuários de uma biblioteca especializada?

O objetivo desta pesquisa é desenvolver uma modelagem que seja capaz de responder a estas questões. Ou seja:

a)Elicitar os critérios a serem utilizados na avaliação das percepções de usuário de uma biblioteca especializada, quanto aos serviços prestados pela mesma

b)Propor uma forma de coleta e tratamento destas percepções e,

c)Coletar e analisar estas percepções em um caso específico.

\section{Materiais e métodos}

Para o alcance dos objetivos foram efetuadas as seguintes etapas: Definição do objeto de estudo; Revisão da literatura para elicitação de critérios e construção do instrumento de coleta; Efetivação da coleta de dados junto a usuários da biblioteca; e, Análise dos dados coletados.

\subsection{Definição do objeto de estudo}

A título de ilustrar a eficácia da proposta, a mesma foi aplicada a uma biblioteca especializada, vinculada a um Instituto de Engenharia. O 
acervo da biblioteca é especializado em Energia Nuclear e abrange as áreas de Física, Química, várias especialidades das Engenharias, Medicina Nuclear e Proteção Radiológica, dentre outras. Ao iniciar este estudo, o acervo era composto de aproximadamente 55.000 documentos entre livros, periódicos, teses, dissertações e relatórios técnicos, porém registrados na base da biblioteca encontravam-se apenas 11.765 documentos.

A biblioteca atende basicamente a três tipos de usuários: diretos, indiretos e externos. São considerados como usuários diretos todos os servidores do Instituto e alunos do Programa de Pós-Graduação deste Instituto. No momento desta pesquisa, a biblioteca contava com 3.522 usuários registrados na rede de bibliotecas da Comissão Nacional de Energia Nuclear (CNEN), sendo que 102 eram usuários diretos.

\subsection{Revisão da literatura para elicitação de critérios e construção do instrumento de coleta}

O conjunto de critérios utilizados na formulação do questionário aplicado aos respondentes é sustentado por uma revisão na literatura efetuada de forma sistematizada e cuja estrutura se baseou nos trabalhos de Costa (2010), Méxas, Quelhas e Costa (2012), Neves, Pereira e Costa, (2015) e Rodríguez, Costa e Do Carmo (2013), sendo que os artigos selecionados foram analisados a partir de pesquisa bibliométrica realizada nas bases SciELO, Scopus e ISI Web of Sciences.

$\mathrm{Na}$ base SciELO os artigos foram identificados a partir da seguinte frase de pesquisa: (Avaliação de bibliotecas OR Avaliação de serviços de bibliotecas OR Serviços de bibliotecas OR Avaliação de serviços de informação OR Avaliação da qualidade de bibliotecas OR Library evaluation OR Quality evaluation of libraries OR Quality of libraries OR Quality of libraries services). Não foi inserida restrição quanto ao período da publicação, tendo sido considerado todo o acervo disponível à época da pesquisa, a qual foi realizada em janeiro de 2012.

Já nas bases Scopus e ISI Web of Science utilizou-se a frase: (Quality Evaluation of Libraries AND Quality of Libraries Services). Com relação aos recortes temporais, a pesquisa foi efetuada nas bases de dados Scopus em fevereiro de 2012 e ISI Web of Science em março de 2012, considerando artigos publicados em periódicos, no período compreendido entre os anos de 2008 a 2012.

Os resumos referentes aos registros indicados pela pesquisa foram analisados originando a seleção de um conjunto de artigos considerados como os mais aderentes à pesquisa em questão. A partir da leitura dos artigos selecionados, elaborou-se o instrumento para a coleta de dados. 0 Quadro 1 exibe os aspectos avaliados a partir do questionário, assim como a codificação dos mesmos, a qual é utilizada como referência na compilação e tabulação dos dados coletados. Este quadro também indica em sua última coluna as referências nos quais os critérios foram inspirados. 


\section{Quadro 1 - Descrição da codificação utilizada na compilação e tabulação} de dados

\begin{tabular}{|c|c|c|c|}
\hline Dimensão & Critério & Código & Referências \\
\hline \multirow{8}{*}{ Acervo } & Adequação & AC 1 & $\begin{array}{l}\text { Suaiden (2000); Coleta e Rozenfeld (2007); } \\
\text { Babalhavaeji et al. (2010); Hariri e Norouzi (2011); } \\
\text { Huang, Huang e Chen (2009) }\end{array}$ \\
\hline & Atualização & AC 2 & $\begin{array}{l}\text { Guimarães et al. (2007); Coleta e Rozenfeld (2007); } \\
\text { Babalhavaeji et al. (2010) }\end{array}$ \\
\hline & Cobertura Nacional & AC 3 & Garcez e Rados (2002); Babalhavaeji et al. (2010) \\
\hline & Cobertura Internacional & AC 4 & $\begin{array}{l}\text { Garcez e Rados (2002); Coleta e Rozenfeld (2007); } \\
\text { Babalhavaeji et al. (2010) }\end{array}$ \\
\hline & Conservação & AC 5 & Cruz et al. (2003); Elizalde (2009) \\
\hline & Conteúdo Informacional & AC 6 & $\begin{array}{l}\text { Alarcón Echenique et al. (2007); Mesquita et al. (2006); } \\
\text { Santos e Andrade (2010) }\end{array}$ \\
\hline & Facilidade de localização & AC 7 & $\begin{array}{l}\text { Madrid (2002); Hariri e Norouzi (2011); e, Huang, } \\
\text { Huang e Chen (2009) }\end{array}$ \\
\hline & Praticidade & AC 8 & $\begin{array}{l}\text { Alarcón Echenique (2007); Huang, Huang e Chen } \\
\text { (2009); Tzeng e Wang (2011) }\end{array}$ \\
\hline \multirow{6}{*}{ Atendimento } & Agilidade no atendimento & AT 1 & $\begin{array}{l}\text { Zamudio Igami, Sampaio e Vergueiro (2005); Freitas, } \\
\text { Bolsanello e Viana (2008); Huang et al. (2009); Lane et } \\
\text { al. (2012) }\end{array}$ \\
\hline & $\begin{array}{l}\text { Agilidade na disponibilização da } \\
\text { informação }\end{array}$ & AT 2 & $\begin{array}{l}\text { Garcez e Rados (2002); Freitas, Bolsanello e Viana } \\
\text { (2008); Babalhavaeji et al. (2010); Cho (2010),Huang, } \\
\text { Huang e Chen (2009); Lane et al. (2012) }\end{array}$ \\
\hline & Empatia & AT 3 & $\begin{array}{l}\text { Santos, Fachin e Varvakis (2003); Sampaio et al. } \\
\text { (2004); Cozin e Turrini (2008); Freitas, Bolsanello e } \\
\text { Viana (2008); Chen e Chou (2011); Garibay Gutiérrez e } \\
\text { Figueroa (2010); Huang, Huang e Chen (2009) }\end{array}$ \\
\hline & Flexibilidade & AT 4 & $\begin{array}{l}\text { Garcez e Rados (2002); Santos, Fachin e Varvakis } \\
\text { (2003); Freitas, Bolsanello e Viana (2008); Cho (2010); } \\
\text { Hariri e Norouzi (2011) }\end{array}$ \\
\hline & $\begin{array}{l}\text { Orientação para consulta à } \\
\text { biblioteca }\end{array}$ & AT 5 & $\begin{array}{l}\text { Guimarães et al. (2007); Babalhavaeji et al. (2010); } \\
\text { Huang, Huang e Chen (2009); Lane et al. (2012) }\end{array}$ \\
\hline & Recuperação da informação & AT 6 & $\begin{array}{l}\text { Café, Santos e Macedo (2001); Madrid (2002); } \\
\text { Bohmerwald (2005); Mesquita et al. (2006); } \\
\text { Babalhavaeji et al. (2010); Hariri e Norouzi (2011) }\end{array}$ \\
\hline \multirow{7}{*}{ Infraestrutura } & Condições ambientais & INF 1 & Guimarães et al. (2007); Hariri e Norouzi (2011) \\
\hline & Disponibilidade das instalações & INF 2 & $\begin{array}{l}\text { Guimarães et al. (2007); Babalhavaeji et al. (2010); } \\
\text { Huang, Huang e Chen (2009) }\end{array}$ \\
\hline & Ergonomia & INF 3 & $\begin{array}{l}\text { Café, Santos e Macedo (2001); Huang, Huang e Chen } \\
\text { (2009) }\end{array}$ \\
\hline & Infraestrutura tecnológica & INF 4 & $\begin{array}{l}\text { Elizalde (2009); Babalhavaeji et al. (2010); Hariri e } \\
\text { Norouzi (2011) }\end{array}$ \\
\hline & Instalações físicas & INF 5 & $\begin{array}{l}\text { Zamudio Igami, Sampaio e Vergueiro (2005); Huang, } \\
\text { Huang e Chen (2009) }\end{array}$ \\
\hline & Integração em redes & INF 6 & $\begin{array}{l}\text { Barber et al. (2004); Bohmerwald (2005); e, } \\
\text { Babalhavaeji et al. (2010) } \\
\text { Hariri e Norouzi (2011); Kyrillidou e Cook (2008) }\end{array}$ \\
\hline & Segurança do ambiente & INF 7 & $\begin{array}{l}\text { Cozin e Turrini (2008); Santos e Andrade (2010); } \\
\text { Garibay, Gutiérrez e Figueroa (2010); Hariri e Norouzi } \\
\text { (2011) }\end{array}$ \\
\hline \multirow{3}{*}{ Internet } & "Disseminação da informação & INT 1 & $\begin{array}{l}\text { Marchiori (1997); e, Suaiden (2000); Café, Santos e } \\
\text { Macedo (2001); Bohmerwald (2005) }\end{array}$ \\
\hline & Uso do catálogo & INT 2 & Madrid (2002) \\
\hline & Websites & INT 3 & $\begin{array}{l}\text { Santos e Andrade (2010); Babalhavaeji et al. (2010); } \\
\text { Hariri e Norouzi (2011) }\end{array}$ \\
\hline
\end{tabular}

Fonte: DUARTE (2012).

\subsection{Coleta de dados junto a usuários da biblioteca}

O instrumento de coleta de dados, ou seja, o questionário solicitou aos respondentes duas respostas em relação aos aspectos reportados no Quadro 1: a importância do critério; e, o desempenho da biblioteca no critério. As seguintes opções foram apresentadas aos respondentes para efetuarem a avaliação quanto ao desempenho do desempenho: Muito 
Bom (MB); Bom (B); Nem ruim/Nem bom (NR); Ruim (R); Muito Ruim $(M R) ;$ Não sei/ Não quero responder (NR). Já quanto à importância dos quesitos para um bom desempenho, os respondentes tinham as seguintes opções: Muito Alta (MA); Alta (A); Média (M); Pouca (P); Sem Importância (SI); Não sei/Não quero responder (NR). A decisão de se colocar a opção NR possibilitou identificar a pergunta foi compreendida ou se foi direcionada ao público certo. Um grande número de respostas "NR" indicaria uma pergunta mal formulada ou direcionada a um público que inadequado (sem o conhecimento suficiente ou não possuía autonomia para respondê-la, por exemplo). A escolha desses conjuntos de opções se baseou em Likert (1932), Miller (1954), Nepomuceno, Costa e Shimoda (2010), Nepomuceno e Costa (2012) e Nepomuceno e Costa (2015).

O questionário foi enviado aos usuários da biblioteca do Instituto por e-mail ou entregue pessoalmente. Além destas formas de acesso aos respondentes, os questionários também foram disponibilizados no Balcão de Referência da biblioteca, para que os servidores e os alunos de pósgraduação que habitualmente vão ao setor pudessem respondê-lo. Na ocasião desta pesquisa o Instituto contava em seu quadro com 251 servidores sendo: 35 Pesquisadores, 69 Tecnologistas, 20 Analistas em Ciência e Tecnologia - C\&T, 75 Técnicos, 51 Assistentes em C\&T e 1 Médico. Contava ainda, com 34 alunos matriculados no Programa de PósGraduação em Ciência e Tecnologia Nucleares (PPGIEN). Dentre os servidores, havia 53 doutores, 53 mestres e 30 especialistas. Tomando em consideração que nem todos utilizam a biblioteca, fez-se um levantamento baseado na relação de servidores da unidade administrativa onde se constatou que somente 83 servidores são usuários do setor e utilizam a biblioteca para dar suporte às suas pesquisas. Logo, o universo pesquisado constituiu-se de 117 respondentes, dos quais 83 são servidores e 34 são alunos efetivamente usuários dos serviços oferecidos pela biblioteca. Dentre estes usuários, 94 responderam ao questionário, 0 que resultou em uma taxa de retorno de $80,34 \%$.

\section{Resultados: apresentação e discussão}

Após o recebimento das respostas ao questionário, as mesmas foram compiladas e processadas com vistas à análise para a compreensão e interpretação das respostas dadas pelos usuários. A seguir apresentamse as análises efetuadas a partir da reunião dos dados, por meio de estatística descritiva, quadros e análise de frequência.

Ao analisar as respostas obtidas na dimensão Acervo, compiladas no Quadro 2, observou-se que em todos os critérios a avaliação quanto ao desempenho foi predominantemente positiva, sendo que AC2 (Atualização) e AC4 (Cobertura internacional) foram os dois critérios que obtiveram o desempenho menos positivo, porém foram considerados os de maior importância pelos respondentes, o que indica a premência em promover melhorias nesses quesitos. Os demais critérios foram 
considerados com bom desempenho e alta importância. Observa-se ainda um número muito baixo de respostas NR.

Quadro 2 - Agregação das frequências atribuídas ao desempenho e importância na dimensão Acervo

\begin{tabular}{|c|c|c|}
\hline \multicolumn{3}{|c|}{ Dimensão ACERVO } \\
\hline Critérios & DESEMPENHO & IMPORTÂNCIA \\
\hline$>A C 1$ & $\mathrm{MB}+\mathrm{B}=74 ; \mathrm{N}=15 ; \mathrm{R}+\mathrm{MR}=2 \mathrm{NR}=2$ & $M A+A=70 ; M=14 ; \quad P+S I=0 ; N R=1$ \\
\hline$>\mathrm{AC2}$ & $\mathrm{MB}+\mathrm{B}=52 ; \mathrm{N}=31 ; \mathrm{R}+\mathrm{MR}=7 ; \mathrm{NR}=4$ & $M A+A=73 ; M=13 ; P+S I=1 ; N R=1$ \\
\hline$>A C 3$ & $M B+B=69 ; N=17 ; R+M R=3 ; N R=5$ & $M A+A=65 ; M=17 ; \quad P+S I=4 ; N R=2$ \\
\hline$>\mathrm{AC} 4$ & $M B+B=64 ; N=19 ; R+M R=6 ; N R=3$ & $M A+A=77 ; M=09 ; \quad P+S I=0 ; N R=2$ \\
\hline$>$ AC5 & $\mathrm{MB}+\mathrm{B}=79 ; \mathrm{N}=10 ; \mathrm{R}+\mathrm{MR}=2 ; \mathrm{NR}=3$ & $M A+A=75 ; M=12 ; P+S I=0 ; N R=0$ \\
\hline$>A C 6$ & $\mathrm{MB}+\mathrm{B}=67 ; \mathrm{N}=21 ; \mathrm{R}+\mathrm{MR}=2 ; \mathrm{NR}=4$ & $M A+A=69 ; \quad M=15 ; P+S I=0 ; N R=3$ \\
\hline$>\mathrm{AC7}$ & $M B+B=73 ; N=14 ; R+M R=4 ; N R=3$ & $M A+A=70 ; M=14 ; \quad P+S I=3 ; N R=1$ \\
\hline$\triangle A C 8$ & $M B+B=68 ; N=16 ; R+M R=5 ; N R=4$ & $M A+A=70 ; M=15 ; P+S I=1 ; N R=1$ \\
\hline
\end{tabular}

Fonte: DUARTE (2012).

Conforme observado do Quadro 3, na dimensão Atendimento, o critério AT1 (Agilidade no atendimento) foi o que apresentou maior nível de importância, seguido do critério AT2 (Agilidade na disponibilização da informação). Observa-se que, para estes dois critérios, o desempenho foi considerado acima da média. Os critérios AT3 (Empatia), AT5 (Orientação para consulta à Biblioteca) e AT6 (Recuperação de informação) se equivalem em nível de importância, sendo que o critério AT4 (Flexibilidade) foi o considerado de menor relevância. Nota-se, ainda, que para todos esses critérios, o desempenho foi considerado acima da média, sendo o AT1 (Agilidade no atendimento) e AT3 (Empatia) com o desempenho mais alto e o AT6 (Recuperação da informação) com o desempenho mais baixo. Também nessa dimensão, o número de respostas NR é muito baixo.

Quadro 3 - Agregação das frequências atribuídas ao desempenho e importância na dimensão Atendimento

\begin{tabular}{|c|c|c|}
\hline \multicolumn{3}{|c|}{ Dimensão ATENDIMENTO } \\
\hline Critérios & DESEMPENHO & IMPORTÂNCIA \\
\hline$>$ AT1 & $\mathrm{MB}+\mathrm{B}=94 ; \quad \mathrm{N}=0 ; \mathrm{R}+\mathrm{MR}=0 ; \mathrm{NR}=0$ & $\mathrm{MA}+\mathrm{A}=87 ; \mathrm{M}=0 ; \mathrm{P}+\mathrm{SI}=0 ; \mathrm{NR}=0$ \\
\hline$>$ AT2 & $\mathrm{MB}+\mathrm{B}=91 ; \mathrm{N}=03 ; \mathrm{R}+\mathrm{MR}=0 ; \mathrm{NR}=0$ & $M A+A=86 ; M=01 ; P+S I=0 ; N R=0$ \\
\hline$>$ AT3 & $\mathrm{MB}+\mathrm{B}=93 ; \mathrm{N}=0 ; \mathrm{R}+\mathrm{MR}=0 ; \mathrm{NR}=0$ & $M A+A=80 ; M=08 ; P+S I=0 ; N R=0$ \\
\hline$>$ AT4 & $\mathrm{MB}+\mathrm{B}=90 ; \mathrm{N}=02 ; \mathrm{R}+\mathrm{MR}=03 ; \mathrm{NR}=0$ & $M A+A=73 ; M=12 ; P+S I=0 ; N R=0$ \\
\hline$>$ AT5 & $\mathrm{MB}+\mathrm{B}=88 ; \mathrm{N}=05 ; \mathrm{R}+\mathrm{MR}=0 ; \mathrm{NR}=1$ & $M A+A=78 ; \quad M=09 ; \quad P+S I=0 ; N R=1$ \\
\hline$>$ AT6 & $M B+B=84 ; N=03 ; R+M R=0 ; N R=5$ & $\mathrm{MA}+\mathrm{A}=79 ; \mathrm{M}=04 ; \mathrm{P}+\mathrm{SI}=0 ; \mathrm{NR}=4$ \\
\hline
\end{tabular}

Fonte: DUARTE (2012).

Quanto à dimensão Infraestrutura o Quadro 4 mostra que o critério INF6 (Integração em redes) foi o avaliado como sendo o de maior importância e desempenho. Embora o critério INF7 (Segurança do ambiente) esteja imediatamente abaixo do critério anterior no item desempenho, sua importância foi considerada apenas alta. Por outro lado, os critérios INF1 (Condições ambientais), INF2 (Disponibilidade das 
instalações), INF3 (Ergonomia), INF4 (Infraestrutura tecnológica) e INF5 (Instalações físicas), mesmo sendo considerados de importância muito alta obtiveram uma frequência de desempenho ruim ou muito ruim bem elevada, sendo o INF3 (Ergonomia), considerado pelos usuários o de pior desempenho. Nesta dimensão, o número de respostas "NR" manteve-se muito baixo, sendo que esta reposta não ocorreu no contexto da avaliação do desempenho da biblioteca.

Quadro 4 - Agregação das frequências atribuídas ao desempenho e importância na dimensão Infraestrutura

\begin{tabular}{c||c|c}
\hline \multicolumn{3}{c}{ Dimensão INFRAESTRUTURA } \\
\hline \multicolumn{1}{c||}{ Critérios } & DESEMPENHO & IMPORTÂNCIA \\
\hline \hline$>$ INF1 & $\mathrm{MB}+\mathrm{B}=58 ; \mathrm{N}=24 ; \mathrm{R}+\mathrm{MR}=10 ; \mathrm{NR}=1$ & $\mathrm{MA}+\mathrm{A}=72 ; \mathrm{M}=15 ; \mathrm{P}+\mathrm{SI}=0 ; \mathrm{NR}=1$ \\
\hline$>\mathrm{INF} 2$ & $\mathrm{MB}+\mathrm{B}=61 ; \mathrm{N}=22 ; \mathrm{R}+\mathrm{MR}=10 ; \mathrm{NR}=0$ & $\mathrm{MA}+\mathrm{A}=73 ; \mathrm{M}=13 ; \mathrm{P}+\mathrm{SI}=0 ; \mathrm{NR}=0$ \\
\hline$>\mathrm{INF3}$ & $\mathrm{MB}+\mathrm{B}=42 ; \mathrm{N}=35 ; \mathrm{R}+\mathrm{MR}=12 ; \mathrm{NR}=3$ & $\mathrm{MA}+\mathrm{A}=68 ; \mathrm{M}=13 ; \mathrm{P}+\mathrm{SI}=4 ; \mathrm{NR}=2$ \\
\hline$>\mathrm{INF} 4$ & $\mathrm{MB}+\mathrm{B}=42 ; \mathrm{N}=30 ; \mathrm{R}+\mathrm{MR}=11 ; \mathrm{NR}=1$ & $\mathrm{MA}+\mathrm{A}=75 ; \mathrm{M}=12 ; \mathrm{P}+\mathrm{SI}=1 ; \mathrm{NR}=0$ \\
\hline$>\mathrm{INF5}$ & $\mathrm{MB}+\mathrm{B}=48 ; \mathrm{N}=33 ; \mathrm{R}+\mathrm{MR}=11 ; \mathrm{NR}=1$ & $\mathrm{MA}+\mathrm{A}=72 ; \mathrm{M}=14 ; \mathrm{P}+\mathrm{SI}=1 ; \mathrm{NR}=0$ \\
\hline$>\mathrm{INF6}$ & $\mathrm{MB}+\mathrm{B}=74 ; \mathrm{N}=14 ; \mathrm{R}+\mathrm{MR}=01 ; \mathrm{NR}=5$ & $\mathrm{MA}+\mathrm{A}=78 ; \mathrm{M}=7 ; \mathrm{P}+\mathrm{SI}=0 ; \mathrm{NR}=2$ \\
\hline$>\mathrm{INF7}$ & $\mathrm{MB}+\mathrm{B}=73 ; \mathrm{N}=12 ; \mathrm{R}+\mathrm{MR}=01 ; \mathrm{NR}=8$ & $\mathrm{MA}+\mathrm{A}=64 ; \mathrm{M}=17 ; \mathrm{P}+\mathrm{SI}=1 ; \mathrm{NR}=6$ \\
\hline
\end{tabular}

Fonte: DUARTE (2012).

Conforme os dados do Quadro 5, na dimensão Internet obteve-se um bom resultado quanto ao desempenho nos três critérios analisados embora tenham sido observadas, em pequeno número, algumas avaliações negativas quanto ao desempenho neste quesito. Percebe-se ainda que o critério INT1 (Disseminação da informação) foi o que apresentou maior importância e também o melhor desempenho, ao passo que o critério com desempenho menos satisfatório, INT2 (Uso de catálogo), foi o que apresentou menor importância. Nessa dimensão não houve respostas "NR" quanto à avaliação do desempenho, tendo ocorrido em um número muito baixo quando da avaliação da importância dos quesitos.

Quadro 5 - Agregação das frequências atribuídas ao desempenho e a importância na dimensão Internet

\begin{tabular}{c||c||c}
\hline \multicolumn{3}{c}{ Dimensão INTERNET } \\
\hline Critérios & DESEMPENHO & IMPORTÂNCIA \\
\hline$>$ INT1 & $\mathrm{MB}+\mathrm{B}=66 ; \mathrm{N}=20 ; \mathrm{R}+\mathrm{MR}=02 ; \mathrm{NR}=5$ & $\mathrm{MA}+\mathrm{A}=79 ; \mathrm{M}=03 ; \mathrm{P}+\mathrm{SI}=0 ; \mathrm{NR}=3$ \\
\hline$>$ INT2 & $\mathrm{MB}+\mathrm{B}=60 ; \mathrm{N}=20 ; \mathrm{R}+\mathrm{MR}=03 ; \mathrm{NR}=10$ & $\mathrm{MA}+\mathrm{A}=68 ; \mathrm{M}=09 ; \mathrm{P}+\mathrm{SI}=02 ; \mathrm{NR}=5$ \\
\hline$>$ INT3 & $\mathrm{MB}+\mathrm{B}=59 ; \mathrm{N}=19 ; \mathrm{R}+\mathrm{MR}=03 ; \mathrm{NR}=10$ & $\mathrm{MA}+\mathrm{A}=71 ; \mathrm{M}=07 ; \mathrm{P}+\mathrm{SI}=01 ; \mathrm{NR}=5$ \\
\hline Fon
\end{tabular}

Fonte: DUARTE (2012).

Como síntese da análise cruzada observa-se que os critérios AC2 (Atualização) e AC4 (Cobertura internacional), ambos associados ao acervo, são os que devem ser priorizados por terem sido considerados de maior importância e apresentarem o desempenho menos satisfatório. 


\section{Conclusão}

Com base na revisão da literatura nacional e internacional, este trabalho permitiu identificar os principais critérios utilizados, para a avaliação no âmbito da qualidade dos serviços oferecidos por bibliotecas e adotar os que mais se adaptavam à biblioteca do Instituto, nas dimensões: acervo, atendimento, infraestrutura, internet e avaliação.

O questionário construído permitiu a captação das percepções dos usuários. A amostra analisada foi representativa do quadro de servidores e alunos usuários dos serviços oferecidos pela biblioteca do Instituto. A taxa de retorno de $80,34 \%$ foi considerada altamente significativa.

Observou-se que o critério melhor avaliado no grau de desempenho foi AC5 (Conservação) na dimensão Acervo, seguido do AC1 (Adequação) e do AC7 (Facilidade de localização). Por outro lado, os aspectos com pior desempenho foram AC2 (Atualização) e AC4 (Cobertura internacional).

Quanto à importância identificou-se que quase todos os aspectos foram considerados predominantemente como sendo de importância pelo menos alta, o que indica o acerto na escolha do conjunto de critérios.

A dimensão Atendimento foi a que obteve avaliação mais alta tanto para o desempenho quanto para a importância na percepção dos respondentes, demonstrando aprovação do atendimento pelos usuários. Isto indica que a biblioteca tem um atendimento de alta qualidade.

A dimensão Internet embora tenha sido bem considerada, foi a que obteve a avaliação mais baixa quanto ao desempenho e a que apresentou importância mais alta.

Considerando os resultados obtidos sugere-se aos gestores da organização que priorizem ações de melhoria na dimensão Acervo. Como exemplo de ação, sugere-se um maior investimento visando à atualização indispensável para dar suporte às pesquisas e projetos da instituição, como também a ampliação na cobertura internacional do acervo. Deverá também ser observada a conformidade quanto à adequação do espaço físico, do mobiliário quanto ao ajuste ergonomicamente adequado às necessidades das pessoas.

Como sugestão para trabalhos futuros recomenda-se o tratamento dos dados neste estudo com técnicas de análise multicritério ou de lógica fuzzy para tratamento dos dados objetivando a extração de mais conhecimento da base de respostas construída na pesquisa,.

\section{Referências}

ALARCÓN ECHENIQUE, M. et al. Evaluación y calidad de los servicios de información: una propuesta teórico-metodológica. ACIMED, v. 15, n. 4, Apr. 2007.

BABALHAVAEJI, F. et al. Quality assessment of academic libraries' performance with a special reference to information technology-based services: suggesting and evaluation checklist. Electronic Library, v. 28, n. 4, p. 592-621, 2010. 
BARBER, E. et al. La automatización y los servicios de las bibliotecas de acceso público de la ciudad autónoma de Buenos Aires y sus alrededores en el marco de la sociedad de la información. Información, Cultura y Sociedad, n. 11, p. 9-56, Dec. 2004.

BOHMERWALD, P. Uma proposta metodológica para avaliação de bibliotecas digitais: usabilidade e comportamento de busca por informação na Biblioteca Digital da PUC-Minas. Ciência da Informação, v. 34, n. 1, p. 95-103, jan. 2005.

CAFÉ, L.; SANTOS, C. dos; MACEDO, F. Proposta de um método para escolha de software de automação de bibliotecas. Ciência da Informação, v. 30, n. 2, p. 70-79, ago. 2001.

CHEN, Y.-T.; CHOU, T.-Y. Applying GRA and QFD to improve library service quality. Journal of Academic Librarianship, v. 37, n. 3, p. 237-245, May 2011.

$\mathrm{CHO}$, J. Developing a SCM-based evaluation system for the Korean academic library consortium. Libri, v. 60, n. 4, p. 321-330, Dec. 2010.

COLETTA, T. das G.; ROZENFELD, H. Indicadores de desempenho para bibliotecas universitárias: definições e aplicações sob o ponto de vista da literatura. Perspectivas em Ciência da Informação, v. 12, n. 3, p. 129 141, set./dez. 2007.

COSTA, H. G. Modelo para Webibliomining: proposta e caso de aplicação. Revista da $F A E$, v. 13, p. 115-125, 2010.

COZIN, S. K.; TURRINI, R. N. T. Evaluation of products and services of a nursing library: user satisfaction. Revista Latino-Americana de Enfermagem, v. 16, n. 4, p. 758-764, Aug. 2008.

CRUZ, A. A. C. da et al. Impacto dos periódicos eletrônicos em bibliotecas universitárias. Ciência da Informação, v. 32, n. 2, p. 47-53, Aug. 2003.

DUARTE, Maria Bernarda Teixeira. Avaliação de serviços oferecidos por bibliotecas: o caso da Biblioteca do Instituto de Engenharia Nuclear - IEN. 2012. 117f. Dissertação (Mestrado em Sistemas de Gestão) Universidade Federal Fluminense, Niterói - RJ, 2012.

ELIZALDE, E. E. Estándares para el Sistema de Bibliotecas de la Universidad de Buenos Aires. Información, Cultura y Sociedad, n. 20, p. 111-116, Jun. 2009.

FREITAS, A. L. P.; BOLSANELLO, F. M. C.; VIANA, N. R. N. G. Avaliação da qualidade de serviços de uma biblioteca universitária: um estudo de caso utilizando o modelo Servqual. Ciência da Informação, v. 37, n. 3, p. 88$112,2008$.

GARCEZ, E. M. S.; RADOS, G. J. V. Necessidades e expectativas dos usuários na educação à distância: estudo preliminar junto ao Programa de Pós-Graduação em Engenharia de Produção da Universidade Federal de Santa Catarina. Ciência da Informação, v. 31, n. 1, p. 13-26, jan. 2002. 
GARIBAY, C. ; GUTIÉRREZ, H. ; FIGUEROA, A. Evaluation of a digital library by means of quality function deployment (QFD) and the Kano model. Journal of Academic Librarianship, v. 36, n. 2, p. 125-132, 2010.

GUIMARÃES, M. C. S. et al. Indicadores de desempenho de bibliotecas no campo da saúde: relato de estudo piloto na FIOCRUZ. Perspectivas em Ciência da Informação, v. 12, n. 1, p. 84-96, jan./abr. 2007.

HARIRI, N.; NOROUZI, Y. Determining evaluation criteria for digital libraries' user interface: a review. Electronic Library, v. 29, n. 5, p. 698722, 2011.

HUANG, T.-T.; HUANG, S.-Y.; CHEN, Y.-H. Applying Fuzzy model on the evaluation of library service quality. IEEE Computer Society, v. 1, p. 7377, 2009.

KYRILLIDOU, M.; COOK, C. The evolution of measurement and evaluation of libraries: a perspective from the Association of Research Libraries. Library Trends, v. 56, n. 4, 2008.

LANE, F. C. et al. Factorial invariance of LibQual $+\AA$ as a measure of library service quality over time. Library \& Information Science Research, v. 34, p. 22-30, 2012.

LIKERT, R.A. Technique for measurement of attitudes. Archives of Psychology, v. 140, n.1, p. 5-55, 1932.

MADRID, I. Evaluación de bibliotecas: su necesidad e importancia. Información, Cultura y Sociedad, n. 6, p.103-113, Jun. 2002.

MARCHIORI, P. Z. "Ciberteca" ou biblioteca virtual: uma perspectiva de gerenciamento de recursos de informação. Ciência da Informação, v. 26, n. 2, maio 1997.

MESQUITA, Rosa et al. Elaboração e aplicação de instrumentos para avaliação da base de dados Scopus. Perspectivas em Ciências da Informação, v. 11, n. 2, p. 187-205, ago. 2006.

MÉXAS, M. P.; QUELHAS, O.; COSTA, H. G. Prioritization of enterprise resource planning systems criteria: Focusing on construction industry. International Journal of Production Economics, v. 139, n. 1, p. 340-350, 2012.

MILLER, G. A. The magical number seven, plus or minus two: some limits on our capacity for processing information. Psychological Review, v. 101, n. 2, p. 343-352, 1954.

NEPOMUCENO, L. D. de O.; COSTA, H. G. Mapeamento de percepções na avaliação dos impactos do mestrado profissional no perfil do seu egresso. Production, v. 22, n. 3, p. 865-873, 2012.

NEPOMUCENO, L. D. de O.; COSTA, H. G.; SHIMODA, E. Impacto do mestrado profissional no desempenho dos seus egressos: intercomparação entre as percepções de discentes, docentes, 
coordenadores e empresa. Gestão \& Produção, v.17, n. 4, p. 817-828, 2010.

NEPOMUCENO, L. D. de O.; COSTA, H. G. Analyzing perceptions about the influence of a master course over the professional skills of its alumni: a multicriteria approach. Pesquisa Operacional, v. 35, n. 1, p. 187-211, Apr. 2015.

NEVES, R. B.; PEREIRA, V.; COSTA, H. G. Auxílio multicritério à decisão aplicado ao planejamento e gestão na indústria de petróleo e gás. Production, v. 25, n. 1, p. 45-53, 2010.

RODRÍGUEZ, D. S. S.; COSTA, H. G.; DO CARMO, L. F. R. R. S. Métodos de auxílio multicritério à decisão aplicados a problemas de PCP: Mapeamento da Producão em periódicos publicados no Brasil. Gestão \& Produção, v. 20, n. 1, p. 134-146, 2013.

SAMPAIO, M. I. C. et al. PAQ - Programa da avaliação da qualidade de produtos e serviços de informação: uma experiência no SIBi/USP. Ciência da Informação, v. 33, n. 1, p. 142-148, Apr. 2004.

SANTOS, A.; ANDRADE, A. Portais de bibliotecas sistemas de avaliação de qualidade dos serviços. Información, Cultura y Sociedad, n. 22, p. 99-118, jun. 2010.

SANTOS, L. C.; FACHIN, G. R. B.; VARVAKIS, G. Gerenciando processos de serviços em bibliotecas. Ciência da Informação, v. 32, n. 2, p. 85-94, ago. 2003.

SUAIDEN, E. J. A biblioteca pública no contexto da sociedade da informação. Ciência da Informação, v. 29, n. 2, p. 52-60, ago. 2000.

TZENG, S.-Y.; WANG, T.-H. Development of an evaluation model for the design of sign systems for university libraries. Journal of Asian Architecture and Building Engineering, v. 10, n. 1, p. 109-116, May 2011.

ZAMUDIO IGAMI, M. P.; SAMPAIO, M. I. C.; VERGUEIRO, W. de C. S. EI uso del Servqual en la verificación de la calidad de los servicios de unidades de información: el caso de la biblioteca del IPEN. Revista Interamericana de Bibliotecología, v. 28, n. 2, p. 177-191, 2005. 